\title{
The single-assignment hub covering problem: Models and linearizations
}

\section{B Y Kara \& B C Tansel}

To cite this article: B Y Kara \& B C Tansel (2003) The single-assignment hub covering problem: Models and linearizations, Journal of the Operational Research Society, 54:1, 59-64, DOI: $10.1057 /$ palgrave.jors. 2601473

To link to this article: https://doi.org/10.1057/palgrave.jors.2601473

曲 Published online: 21 Dec 2017.

Submit your article to this journal $\pi$

Џ Article views: 4

Citing articles: 5 View citing articles $₫$ 


\title{
The single-assignment hub covering problem: Models and linearizations
}

\author{
BY Kara and BC Tansel* \\ Department of Industrial Engineering, Bilkent University, Ankara, Turkey
}

We study the hub covering problem which, so far, has remained one of the unstudied hub location problems in the literature. We give a combinatorial and a new integer programming formulation of the hub covering problem that is different from earlier integer programming formulations. Both new and old formulations are nonlinear binary integer programs. We give three linearizations for the old model and one linearization for the new one and test their computational performances based on 80 instances of the $\mathrm{CAB}$ data set. Computational results indicate that the linear version of the new model performs significantly better than the most successful linearization of the old model both in terms of average and maximum CPU times as well as in core storage requirements.

Journal of the Operational Research Society (2003) 54, 59-64. doi:10.1057/palgrave.jors.2601473

Keywords: hub location; hub cover; location; combinatorial problems; integer programming

\section{Introduction}

Hub location problems arise when it is desirable to consolidate and disseminate flows at certain centralized locations in many-to-many distribution systems. Applications arise in air passenger travel, cargo delivery, and telecommunication network design. ${ }^{1-3}$

The generic problem involves $n$ cities that exchange flows among themselves. The flows may be passengers, cargo, or information packets. Some $p$ of the $n$ cities are taken to be hubs and are used as consolidation and dissemination centers that receive, process, and redistribute flows. The flow from an origin $i$ to a destination $j$ is routed from $i$ to $j$ via a pair of hubs that are assigned to serve these two cities. The hub-to-hub portion of the journey is discounted by a factor $\alpha(0 \leqslant \alpha \leqslant 1)$ to account for economies of scale that result from bulk transportation between hub cities. The core problem involves determining the locations of hubs and the allocation of the demands to hubs. These decisions depend on what criterion is used to optimize the system performance. The primary focus so far has been on the total cost criterion. ${ }^{4-10}$ The first deviation from the total cost criterion seems to be due to O'Kelly and Miller, ${ }^{11}$ where the focus is shifted from the total cost to the minimax criterion for $p=1$. The minimax criterion for general $p$ is studied by Kara and Tansel, ${ }^{12}$ and a new minimax model, called the latest arrival hub location problem, is formulated and analysed by Kara and Tansel. ${ }^{13} \mathrm{~A}$ third criterion, which is mentioned by

*Correspondence: BC Tansel, Department of Industrial Engineering, Bilkent University, Bilkent 06533, Ankara, Turkey.

E-mail: barbaros@bilkent.edu.tr
Campbell, ${ }^{14}$ but which has not yet received any attention in the literature, is the covering criterion, which is the focus of this paper. In both the p-hub median (minisum) and the p-hub center (minimax) problems, the number of hubs, $p$, is fixed and their locations are optimized to minimize the total cost or the worst case cost. In contrast, $p$ is a variable of the problem in the hub covering problem and it is to be minimized while making sure that all trip times between origin/destination pairs are within predetermined bounds. Restricting the travel time to predetermined time bounds is an appropriate constraint for cargo delivery systems where certain deadlines must be met on delivery time. Typical applications arise in overnight package delivery. ${ }^{15}$ Other applications arise in the delivery of time-sensitive or perishable items. The covering model may also be appropriate for passenger flows if it is desired to keep the dissatisfaction of passengers associated with long trip times within reasonable bounds.

The objective of minimizing the number of hubs while obeying a maximum time bound on travel time makes sense in view of the fact that establishing and operating hubs involve large amounts of set-up and operating costs. Alternative objective functions such as the minimization of the total transportation cost or the transportation cost plus fixed charges could also be used subject to time bounds. None of these problems have yet been studied in the literature other than an expository formulation of the hub covering problem given by Campbell. ${ }^{14}$ Our study of the hub covering problem fills a gap in this respect. The insights obtained in the paper for the solution of this problem may also prove useful in the solution of other time constrained problems with different objective functions. 
In the paper, we first give a combinatorial formulation of the hub covering problem and propose a new nonlinear integer programming model. We then give a linearization for the new model and provide three different linearizations for Campbell's earlier quadratic formulation. ${ }^{14}$ The computational merits of these linearizations are tested on 80 instances of the CAB data set. ${ }^{4}$ The CPU times based on the new formulation indicate a significant improvement in computational performance. This demonstrates that different modeling perspectives may indeed make a very substantial difference in computability.

The paper is organized as follows. We first give a combinatorial formulation of the hub covering problem and present a new integer programming formulation. In the same section, we also give an earlier model of Campbell. ${ }^{14}$ We provide three different linearizations for Campbell's model and one linearization for the new model in the second section. In the subsequent section, we report computational results on all the models. The paper ends with concluding remarks.

\section{Problem formulations}

Let $N=\{1, \ldots, n\}$ be the set of cities and let $t_{i j}=t_{j i}$ be the travel time between nodes $i$ and $j$. Assume that $t_{i j}+t_{j k} \geqslant t_{i k} \forall i, j, k$. Let $H \subset N$ be a set of nodes that specify the locations of hubs and denote by $a(i) \in H$ the hub that serves node $i$ (we assume that each node is served by a single hub, e.g., single-assignment). Let $\alpha(0 \leqslant \alpha \leqslant 1)$ be a discount factor for hub-to-hub transportation and let $\beta$ be a predetermined bound that imposes a deadline for travel time between any pair of cities. We refer to $\beta$ as the cover radius. The hub covering problem involves choosing the locations of the minimum number of hubs such that the travel time between any pair of cities is no more than the cover radius. The combinatorial formulation of the problem is:

$$
\min _{H \subset N}|H|
$$

s.t.

$$
t_{i a(i)}+\alpha t_{a(i) a(j)}+t_{a(j) j} \leqslant \beta \quad \forall i, j \in N \quad a(i) \in H \quad \forall i
$$

The recognition form of the problem is NP-Complete ${ }^{12}$ and the optimization form is NP-Hard.

We now propose an integer programming formulation that is directly derivable from the above combinatorial formulation. Let $X_{i k}$ be a binary variable that takes on the value 1 if node $i$ is served from a hub at node $k$ and 0 otherwise. Note that $X_{k k}=1$ iff there is a hub at node $k$. An integer programming formulation of the hub covering problem $(\mathrm{HC})$, is as follows:

$$
\min \sum_{k} X_{k k}
$$

s.t.

$$
\begin{aligned}
\left(t_{i r}+\alpha t_{r k}+t_{j k}\right) X_{i r} X_{j k} & \leqslant \beta & & \forall i, j, k, r \\
\sum_{k} X_{i k} & =1 & & \forall i \in N \\
X_{i k} & \leqslant X_{k k} & & \forall i, k \in N \\
X_{i k} & \in\{0,1\} & & \forall i, k \in N
\end{aligned}
$$

Constraint (1) ensures that the deadline on travel times are met while constraints (2) and (4) ensure that every node is assigned to exactly one hub. Constraint (3) ensures that such an assignment cannot be made unless there is a hub at node $k$. HC is a binary program with $n^{2}$ binary variables and $n^{4}+n^{2}+n$ constraints. The model is nonlinear due to constraint (1).

An earlier formulation of the hub covering problem, which is different from HC, is given by Campbell. ${ }^{14}$ In Campbell's formulation, constraint (1) is replaced by the set covering constraints

$$
\sum_{k, m} V_{i j k m} X_{i k} X_{j m} \geqslant 1 \quad \forall i, j \in N
$$

where the coefficients $V_{i j k m}$ are such that $V_{i j k m}=1$ if $t_{i k}+\alpha t_{k m}+t_{j m} \leqslant \beta$ and 0 otherwise. We refer to this formulation of the problem as the hub set covering (HSC) formulation. Constraints (2), (3), (4), and the objective function in both formulations are the same. Observe that, in HSC, the number of binary variables is $n^{2}$ and the number of constraints is $2 n^{2}+n$.

\section{Linearizations}

We first propose the following linearization for HC. Replace (1) with constraint (6) below:

$$
\left(t_{i r}+\alpha t_{r k}\right) X_{i r}+t_{j k} X_{j k} \leqslant \beta \quad \forall i, j, k, r
$$

We refer to this linearization of HC as HC-Lin.

Theorem 1 Any feasible solution to $\mathrm{HC}$ is a feasible solution to HC-Lin and vice versa.

Proof. Let $\hat{X}$ be a feasible solution to HC. Since constraints (2), (3), and (4) are common to both HC and HCLin, it suffices to show that $\hat{X}$ is feasible to (6). Consider the constraint (6) associated with the quadruplet $i, j, k, r$. There are four cases to consider depending on the values of $\hat{X}_{i r}$ and $\hat{X}_{j k}$.

- Case 1: $\hat{X}_{i r}=1, \hat{X}_{j k}=1$ : Then (1) and (6) give the same left hand sides.

- Case 2: $\hat{X}_{i r}=1, \hat{X}_{j k}=0$ : Then the left side of (6) is $t_{i r}+\alpha t_{r k}$ and we must show that this is less than or equal to $\beta$. Either $\hat{X}_{k k}=1$ or 0 . If $\hat{X}_{k k}=1$, constraint (1) for $i, k, r, k$ yields $t_{i r}+\alpha t_{r k}+t_{k k} \leqslant \beta$, which implies that (6) is satisfied.

If $\hat{X}_{k k}=0$ then (2) implies that there exists an index $l \neq k$ such that $\hat{X}_{k l}=1$. Constraint (1) for $i, k, r, l$ yields 
$t_{i r}+\alpha t_{r l}+t_{l k} \leqslant \beta$ (7). Noting that $0 \leqslant \alpha \leqslant 1$, and that $t_{i r} \leqslant t_{r l}+t_{l k}$, we have $t_{i r}+\alpha t_{r k} \leqslant t_{i r}+\alpha t_{r l}+t_{l k}$, which implies with (7) that (6) is satisfied.

- Case 3: $\hat{X}_{i r}=0, \hat{X}_{j k}=1$ : In this case the left hand side of (6) is $t_{j k}$. Since $\hat{X}_{i r}=0$, constraint (2) implies that there exists an index $l \neq r$ such that $\hat{X}_{i l}=1$. Constraint (1) for $i, j, l, k$ gives $\beta \geqslant t_{i l}+\alpha t_{l k}+t_{j k}$, which implies that $t_{j k} \leqslant \beta$.

- Case 4: $\hat{X}_{i r}=0, \hat{X}_{j k}=0$ : In this case both (1) and (6) yield the same left hand sides.

To prove the converse, observe that the left side of (1) is always less than or equal to the left side of (6), which implies that any feasible solution to HC-Lin is also feasible to $\mathrm{HC}$.

Corollary. An optimum solution to HC-Lin is also an optimum solution to $\mathrm{HC}$ (and vice versa).

HC-Lin is a strong linearization of HC in the sense that: (1) it uses precisely the same set of variables as in $\mathrm{HC}$, that is, there is no change in the dimension of the space, (2) the feasible sets are exactly the same, and (3) the optimal sets are the same. It will be evident later that the same cannot be said for the hub set covering formulation of the problem. The linearizations we give for $\mathrm{HSC}$ require additional variables, which increase the dimension of the space. In this sense, the linearizations of HSC do not seem to be as strong as the linearization we proposed for HC. Computational evidence also suggests that HC-Lin yields significantly faster solution times than all linearizations of HSC.

We now provide three linearizations of HSC. The first one is due to Campbell. ${ }^{14}$ The second is an adaptation of a linearization of Skorin-Kapov et al. ${ }^{8}$ The third is a linearization of ours.

Campbell $^{14}$ linearizes HSC by introducing new variables $X_{i j k m}$ for the product $X_{i k} X_{j m}$. These four indexed variables are also used in the other two linearizations. Campbell's linearization, which we refer to as HSC-Lin1, is as follows:

$$
\begin{gathered}
\min \sum_{k} X_{k k} \\
\text { s.t. (2)-(4) } \\
\sum_{k, m} V_{i j k m} X_{i j k m} \geqslant 1 \quad \forall i, j \\
\sum_{j} \sum_{m}\left(w_{i j} X_{i j k m}+w_{j i} X_{j i m k}\right)=\sum_{j}\left(w_{i j}+w_{j i}\right) X_{i k} \quad \forall i, k \\
X_{i j k m} \in\{0,1\} \quad \forall i, j, k, m
\end{gathered}
$$

In this linearization, $w_{i j} \geqslant 0$ is the annual flow from node $i$ to node $j$.

Next, we give the linearization HSC-Lin2, which is an adaptation of the linearization of Skorin Kapov et al. ${ }^{8}$

$$
\min \sum_{k} X_{k k}
$$

$$
\begin{aligned}
& \text { s.t. (2)-(4), (8) } \\
& \sum_{k} X_{i j k m}=X_{i k} \quad \forall i, j, m \\
& \sum_{m} X_{i j k m}=X_{j m} \quad \forall i, j, k \\
& X_{i j k m} \in\{0,1\} \quad \forall i, j, k, m
\end{aligned}
$$

The third linearization, HSC-Lin3, which is motivated by a linearization used in Kara and Tansel, ${ }^{12}$ is as follows:

$$
\begin{array}{cl}
\min \sum_{k} X_{k k} & \\
\text { s.t. }(2)-(4),(8) & \\
X_{i j k m} \geqslant X_{i k}+X_{j m}-1 & \forall i, j, k, m \\
\sum_{k, m} X_{i j k m}=1 & \forall i, j \\
X_{i j k m} \geqslant 0 & \forall i, j, k, m
\end{array}
$$

The correctness of these linearizations can be directly justified using the fact that $X_{i j k m}=1(=0)$ iff $X_{i k} X_{j m}=$ $1(=0)$. In HSC-Lin3, the integrality constraint on $X_{i j k m}$ is relaxed. This is well justified by the inclusion of the constraints (12) and (13). In HSC-Lin1 and HSC-Lin2 there are $n^{2}+n^{4}$ binary variables, whereas in HSC-Lin3 there are $n^{2}$ binary and $n^{4}$ real variables. There are $3 n^{2}+n$ constraints in HSC-Lin $1,2 n^{3}+2 n^{2}+n$ constraints in HSCLin2, and $n^{4}+3 n^{2}+n$ constraints in HSC-Lin3.

\section{Computational Analysis}

We test all the linearizations with the CAB data set using CPLEX 5.0. This data set is considered to be a benchmark by most researchers in the hub location area. The CAB data set is generated from the Civil Aeronautics Board Survey of 1970 passenger data in the United States. It contains the passenger flows and distances between 25 cities (Figure 1).

In our experimental design, we take $n \in\{10,15,20,25\}$ and $\alpha \in\{0.2,0.4,0.6,0.8,1.0\}$. The cover radius $\beta$ is not a part of the $\mathrm{CAB}$ data set. We choose $\beta$ on the basis of the $\mathrm{p}$-hub center optimal objective function values, denoted by $Z_{p}^{*}$. We generate a total of 80 instances taking $\beta \in\left\{Z_{2}^{*}, Z_{3}^{*}, Z_{4}^{*}, Z_{5}^{*}\right\}$ for each $(\alpha, n)$ combination except for $(\alpha, n)=(1.0,15)$ and $(1.0,20)$. For these two cases, we have $Z_{2}^{*}=2611>Z_{3}^{*}=Z_{4}^{*}=Z_{5}^{*}=2600$. Because the $Z_{p}^{*}$ values coincide for $p=3,4,5$ in these two cases, we take two of the $\beta$ values strictly between $Z_{3}^{*}$ and $Z_{2}^{*}$. The complete list of the $\beta$ values is given in Table 1 .

Table 2 compares the computational performance of the three linearizations of the hub set covering model and the linearization of the hub cover model for different values of $n$ on the basis of three different criteria: number of instances solved, average CPU, and maximum CPU. The time limit to abandon a solution is 8.5 hours. The third column of the table reveals that none of the linearizations of HSC are 


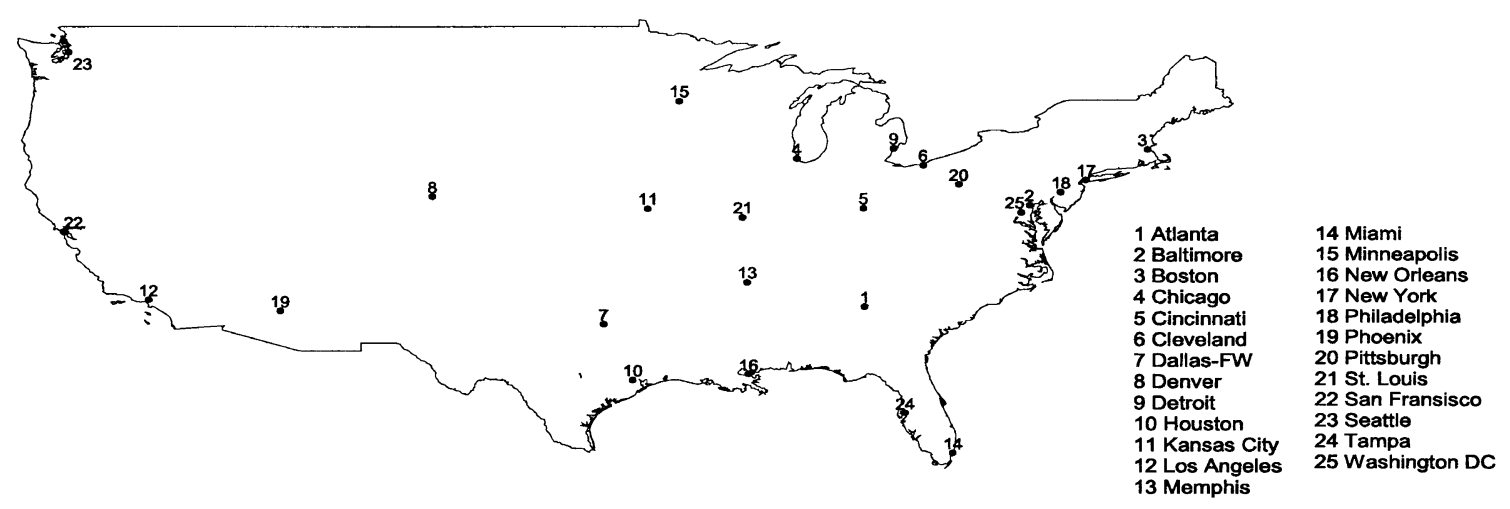

Figure 1 Cities used in the $\mathrm{CAB}$ data set.

successful in solving problems of size $n=25$ while the linearization of HC solves all instances of this size. For the next largest size, $n=20$, HC-Lin solves all instances while its closest competitor, HSC-Lin3, attains a success rate of $25 \%$ for this size. The other two linearizations cannot solve any instances of $n=20$.

Comparing now the four linearizations on the basis of average and maximum CPU times, we see that HSC-Lin3 is the most successful of the three linearizations of HSC. Despite that, its success is completely overshadowed by the far more superior performance of HC-Lin. For example, for $n=20$, the average CPU time of HC-Lin is at least 283 times better than the average CPU time of HSC-Lin3. In terms of the maximum CPU time, HC-Lin is, again, about 50 times faster than HSC-Lin3 for $n=15$. The comparison of HC-Lin and HSC-Lin 3 cannot be made for $n=20$ and 25, because not all instances are solved by HSC-Lin3 for these two sizes. Nevertheless, it would not be unreasonable to expect an even larger gap between their performances with increased $n$.

Table 1 The cover radii used for test problems

\begin{tabular}{|c|c|c|c|c|c|}
\hline & \multicolumn{5}{|c|}{$\alpha$} \\
\hline & 0.2 & 0.4 & 0.6 & 0.8 & 1.0 \\
\hline$n=10$ & $\begin{array}{r}1425 \\
1117 \\
811 \\
736\end{array}$ & $\begin{array}{r}1627 \\
1185 \\
970 \\
863\end{array}$ & $\begin{array}{l}1671 \\
1387 \\
1148 \\
1079\end{array}$ & $\begin{array}{l}1744 \\
1589 \\
1457 \\
1413\end{array}$ & $\begin{array}{l}1839 \\
1791 \\
1770 \\
1766\end{array}$ \\
\hline$n=15$ & $\begin{array}{l}2004 \\
1638 \\
1324 \\
1149\end{array}$ & $\begin{array}{l}2019 \\
1741 \\
1436 \\
1287\end{array}$ & $\begin{array}{l}2103 \\
1844 \\
1756 \\
1560\end{array}$ & $\begin{array}{l}2424 \\
2165 \\
2100 \\
2080\end{array}$ & $\begin{array}{l}2611 \\
2610 \\
2605 \\
2600\end{array}$ \\
\hline$n=20$ & $\begin{array}{l}1851 \\
1549 \\
1356 \\
1162\end{array}$ & $\begin{array}{l}2067 \\
1744 \\
1473 \\
1386\end{array}$ & $\begin{array}{l}2255 \\
1996 \\
1835 \\
1663\end{array}$ & $\begin{array}{l}2493 \\
2264 \\
2154 \\
2118\end{array}$ & $\begin{array}{l}2611 \\
2605 \\
2601 \\
2600\end{array}$ \\
\hline$n=25$ & $\begin{array}{l}2136 \\
1913 \\
1617 \\
1346\end{array}$ & $\begin{array}{l}2401 \\
2099 \\
1881 \\
1597\end{array}$ & $\begin{array}{l}2557 \\
2336 \\
2184 \\
2002\end{array}$ & $\begin{array}{l}2713 \\
2552 \\
2457 \\
2307\end{array}$ & $\begin{array}{l}2826 \\
2762 \\
2726 \\
2725\end{array}$ \\
\hline
\end{tabular}

It is also worth mentioning that, of all the 20 instances solved by HC-Lin for $n=25,17$ are solved in less than 10 minutes while the remaining three instances are solved in 17, 47, and 86 minutes. In fact, of the 17 instances solved under 10 minutes, 13 are solved in less than 5 minutes, and 7 are solved in less than 1 minute.

Some conclusions can also be drawn on the spatial behavior of the optimal hub locations as a function of the parameters $\alpha$ and $\beta$. We use the data set for $n=25$ to illustrate some of our conclusions. First, we set $\alpha=0.4$ and observe the effects of decreased $\beta$ for this $\alpha$. For $\beta=2401$ the optimal solution has two hubs, one at Denver, the other at Cincinnati, with allocations as shown in Figure 2a. When $\beta$ is decreased to 2099, two hubs are no longer sufficient. The hub at Cincinnati splits, resulting in two hubs at Atlanta and at Pittsburgh, while the hub at Denver remains intact (Figure 2b). The allocation set of Denver is still the same while the allocation set of the hub that was formerly at Cincinnati is split now between the two hubs at Atlanta and Pittsburgh. Further reduction of $\beta$ to 1881 results in a splitting of the hub at Denver into two hubs at Los Angeles and Seattle. The hubs at Atlanta and Pittsburgh have moved now to Memphis and Cincinnati (Figure 2c). It can be observed that there is a general westward shift of the hubs, both the ones at Atlanta and Pittsburgh as well as the now-split hub at Denver. Even though the new allocation sets seem to be quite different from the old ones there is quite a bit of structure in the new allocation sets, particularly for the hubs at Memphis (formerly at Atlanta) and Cincinnati (formerly at Pittsburgh). The allocation set of Memphis includes all cities that were formerly served by Atlanta. Likewise the allocation set of Cincinnati includes all cities that were formerly served by Pittsburgh. However, the allocation sets of both Memphis and Cincinnati additionally include new cities that were formerly served by Denver (this is expected since Denver is no longer a hub). Note also that the cities west of Denver are now served by the new hubs at Los Angeles and Seattle. We note in passing that Seattle is on a critical path (a longest path whose length matches the cover radius) for $\beta=2099$ so that it seems natural for it to 
Table 2 Computational comparison of linearizations of HSC and of HC

\begin{tabular}{|c|c|c|c|c|c|}
\hline $\begin{array}{l}\text { Nonlinear } \\
\text { model }\end{array}$ & Linearization & $\mathrm{n}$ & $\begin{array}{l}\text { Instances solved in } \\
8.5 \mathrm{hr} \text { time limit }\end{array}$ & Average $C P U$ & $\operatorname{Max} C P U$ \\
\hline \multirow[t]{12}{*}{ HSC } & HSC-Lin1 & 10 & 20 & $4.3 \mathrm{~s}$ & $11.6 \mathrm{~s}$ \\
\hline & & 15 & 17 & $2.5 \mathrm{hr}$ & $6.5 \mathrm{hr}$ \\
\hline & & 20 & none & - & - \\
\hline & & 25 & none & - & - \\
\hline & HSC-Lin2 & 10 & 20 & $1.45 \mathrm{~min}$ & $3.7 \mathrm{~min}$ \\
\hline & & 15 & 20 & $1.5 \mathrm{hr}$ & $4.5 \mathrm{hr}$ \\
\hline & & 20 & none & - & - \\
\hline & & 25 & none & - & - \\
\hline & HSC-Lin3 & 10 & 20 & $0.99 \mathrm{~min}$ & $2.2 \mathrm{~min}$ \\
\hline & & 15 & 20 & $1.3 \mathrm{hr}$ & $3.4 \mathrm{hr}$ \\
\hline & & 20 & 5 & $\geqslant 8.5 \mathrm{hr}$ & - \\
\hline & & 25 & none & - & - \\
\hline \multirow[t]{4}{*}{$\mathrm{HC}$} & HC-Lin & 10 & 20 & $1 \mathrm{~s}$ & $4.5 \mathrm{~s}$ \\
\hline & & 15 & 20 & $21.5 \mathrm{~s}$ & $4.2 \mathrm{~min}$ \\
\hline & & 20 & 20 & $1.8 \mathrm{~min}$ & $18.4 \mathrm{~min}$ \\
\hline & & 25 & 20 & $10.2 \mathrm{~min}$ & $1.4 \mathrm{hr}$ \\
\hline
\end{tabular}

become a new hub with a reduction in $\beta$. Further reduction of $\beta$ from 1881 to 1597 retains the two hubs at Los Angeles and Seattle intact, together with their allocations (Figure 2d), while the hub at Memphis moves now to Kansas City and the hub at Cincinnati splits and moves eastward to two new hubs at Miami and Philadelphia. The allocation sets associated with these hubs are now reconfigured accordingly.

To see the effects of $\alpha$, we vary $\alpha$ from 0.2 to 1.0 while trying to keep the minimum number of hubs at two. This, in general, requires the use of increased cover radius with increased $\alpha$. For example, as $\alpha$ is increased from 0.2 to 0.4 ,
$0.6,0.8$, and $1.0, \beta$ has to be increased from 2136 to 2401 , $2597,2713,2826$, respectively, to keep the minimum number of hubs $p=2$. There seems to be a tendency for the hubs to get closer with increased $\alpha$ (accompanied with increased $\beta$ ) if the minimum number of hubs is two. The behaviour of hubs in this respect is irregular when the minimum number of hubs is larger than two (e.g., one pair of hubs may get closer while another pair may get farther apart).

Models of medium size can thus be solved optimally within reasonable CPU time using our linearization HC-Lin. Although heuristic methods or simulation are other possible

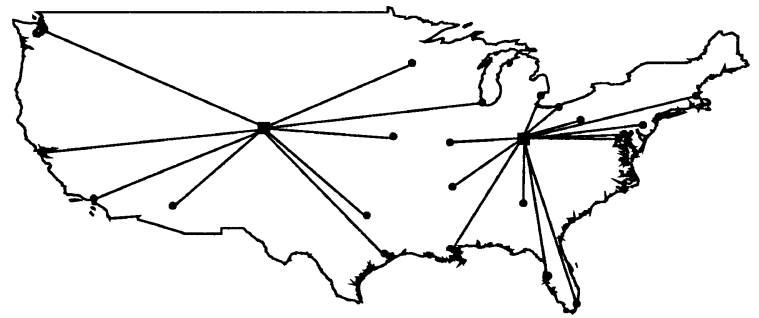

a) Beta $=\mathbf{2 4 0 1}$

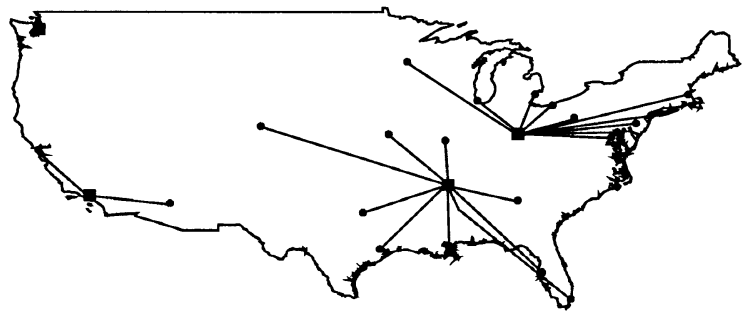

c) Beta $=1881$

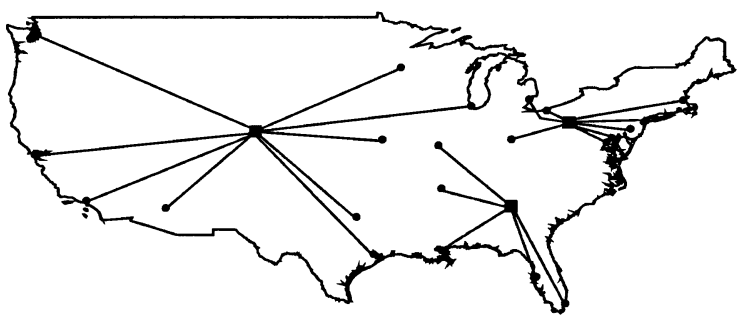

b) Beta $=2099$

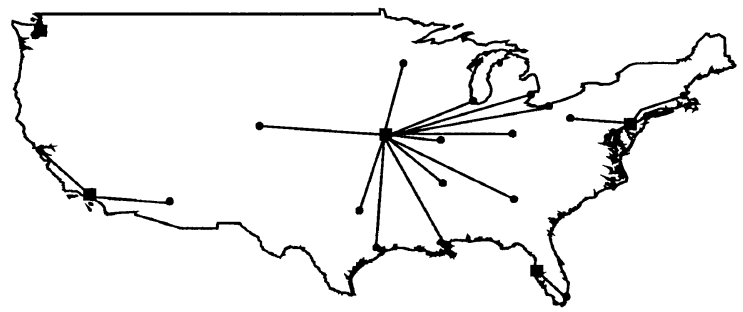

d) Beta $=1597$

Figure 2 Optimal hub locations and allocations for $\alpha=0.4$ and (a) $\beta=2401$, (b) $\beta=2099$, (c) $\beta=1881$, and (d) $\beta=1597$. 
tools of analysis for such problems, they do not necessarily find optimal solutions. The worst case optimality gap may be quite large. For larger sized problems where finding an optimal solution may require excessive CPU times, the use of heuristics or simulation may be unavoidable. Additionally, simulation may be useful for handling more complicated structures in distribution systems that involve day-to-day decisions such as routing and scheduling.

\section{Conclusion}

In this paper, we study the hub covering problem that has remained so far as one of the unstudied hub problems in the literature. The common practice in delivery of time-sensitive items is to increase resources as necessary (e.g., number of hubs, fleet size, technologically improved fleet composition for faster delivery) to meet imposed deadlines. This may be more costly than necessary. The model studied in the paper gives optimal solutions with which the existing systems can be compared.

A computational study based on 80 instances generated from the $\mathrm{CAB}$ data set is carried out in the paper to test the computational performance of three different linearizations of an initial model provided by Campbell ${ }^{14}$ and a linearized new model proposed in this paper. The computational tests indicate that the linearization of the new model's performance is significantly better than the linearizations of the old model. We note also that the new linear model results in a binary program with $n^{2}$ binary variables while two of the linearizations of the earlier model involve $n^{2}+n^{4}$ binary variables and its third linearization involves $n^{2}$ binary and $n^{4}$ real variables. This shows that there are also substantial reductions in core storage requirements in favor of the new model.

The problem solved in the paper is a static problem. This is well justified when transport distances of concern are over relatively large geographical regions. Regardless of the mode of transportation (air or ground), travel times over large distances are relatively non-varying over time. Hence, the static model appears to handle most real world situations where the demand cities are spread over large territories. If dynamic factors are such that variation over time is nonnegligible (e.g., travel through congested metropolitan areas), then choosing the optimum locations of minimum number of hubs while obeying time bounds with timedependent travel times can be converted to the static problem by defining a time matrix $\bar{T}=\left[\bar{t}_{i j}\right]$ where $\bar{t}_{i j}=$ $\max _{0 \leqslant \tau \leqslant \tau^{*}} t_{i j}(\tau)$ with $t_{i j}(\tau)$ representing the travel time between points $i$ and $j$ at time $\tau$ ( $\tau^{*}$ is the planning horizon). This reduces the family of constraints

$t_{i a(i)}(\tau)+\alpha t_{a(i) a(j)}(\tau)+t_{a(j) j}(\tau) \leqslant \beta \quad \forall i, j, \quad 0 \leqslant \tau \leqslant \tau^{*}$

to the finite family

$$
\bar{t}_{i a(i)}+\alpha \bar{t}_{a(i) a(j)}+\bar{t}_{a(j) j} \leqslant \beta \quad \forall i, j .
$$

Stochastic variations on travel times can also be handled in a similar way by choosing the worst realization for each pair of cities. This can all be well justified under the assumption that the hub locations and allocations remain fixed throughout the planning horizon. Changing the locations of hubs in each year is not an easy matter because of various managerial and economic considerations. Consequently, the assumption of non-varying hub locations is a reasonable assumption. The allocation decisions may be more easily modified from period to period. If such is the case then this can be reflected into the model by appending an additional time index to the allocation variables. This would increase the model size by a multiplicative factor of the number of time periods. Consequently, such problems can be formulated as integer programs if time is discretized, but solution times may be prohibitive unless special techniques are developed for handling much larger sized problems.

\section{References}

1 Toh RS and Higgins RC (1985). The impact of hub and spoke network centralization and route monopoly on domestic airline profitability. Transport $J$ 24: 16-27.

2 Kuby MJ and Gray RG (1993). The hub network design problem with stopover and feeders: The case of Federal Express. Transport Res A-Pol 27A: 1-12.

3 Klincewicz JG (1998). Hub location in backbone/tributary network design: A review. Location Sci 6: 307-335.

4 O'Kelly ME (1986). The location of interacting hub facilities. Transport Sci 20: 92-105.

5 O'Kelly, ME (1987). A quadratic integer program for the location of interacting hub facilities. Eur $J$ Opl Res 32: 393-404.

6 Aykin T (1995). The hub location and the routing problem. Eur J Opl Res 83: 200-219.

7 Campbell JF (1996). Hub location and the p-hub median problem. Opns Res 44: 923-935.

8 Skorin-Kapov D, Skorin-Kapov J and O'Kelly ME (1996). Tight linear programming relaxations of uncapacitated p-hub median problems. Eur J Opl Res 94: 582-593.

9 Ernst AT and Krishnamoorthy M (1996). Efficient algorithms for the uncapacitated single allocation p-hub median problem. Location Sci 4: 139-154.

10 Ernst AT and Krishnamoorthy M (1998). Exact and heuristic algorithms for the uncapacitated multiple allocation p-hub median problem. Eur J Opl Res 104: 100-112.

11 O'Kelly ME and Miller HJ (1991). Solution strategies for the single facility minimax hub location problem. Pag Reg Sci 70: 393-404.

12 Kara BY and Tansel BC (2000). On the single-assignment p-hub center problem. Eur J Opl Res 125: 648-655.

13 Kara BY and Tansel BC (2001). The latest arrival hub location problem. Mngt Sci 47: 1408-1420.

14 Campbell JF (1994). Integer programming formulations of discrete hub location problems. Eur J Opl Res 72: 387-405.

15 Sigafoos RA and Easson RR (1988). Absolutely Positively Overnight! The Case of Federal Express. St Lukes Press: Memphis.

Received August 2001; accepted July 2002 after one revision 